\title{
Cancer-Associated Immune Resistance and Evasion of Immune Surveillance in Colorectal Cancer
}

\author{
Pietro Parcesepe, ${ }^{1}$ Guido Giordano, ${ }^{2}$ Carmelo Laudanna, ${ }^{3}$ \\ Antonio Febbraro, ${ }^{2}$ and Massimo Pancione ${ }^{4}$ \\ ${ }^{1}$ Department of Pathology and Diagnostics, University of Verona, 31134 Verona, Italy \\ ${ }^{2}$ Medical Oncology Unit, Fatebenefratelli Hospital, 82100 Benevento, Italy \\ ${ }^{3}$ Department of Experimental and Clinical Medicine "Gaetano Salvatore", University "Magna Grecia", 88100 Catanzaro, Italy \\ ${ }^{4}$ Department of Sciences and Technologies, University of Sannio, 82100 Benevento, Italy
}

Correspondence should be addressed to Massimo Pancione; massimo.pancione@unisannio.it

Received 30 October 2015; Revised 11 January 2016; Accepted 20 January 2016

Academic Editor: Hassan Ashktorab

Copyright (C) 2016 Pietro Parcesepe et al. This is an open access article distributed under the Creative Commons Attribution License, which permits unrestricted use, distribution, and reproduction in any medium, provided the original work is properly cited.

Data from molecular profiles of tumors and tumor associated cells provide a model in which cancer cells can acquire the capability of avoiding immune surveillance by expressing an immune-like phenotype. Recent works reveal that expression of immune antigens (PDL1, CD47, CD73, CD14, CD68, MAC387, CD163, DAP12, and CD15) by tumor cells "immune resistance," combined with prometastatic function of nonmalignant infiltrating cells, may represent a strategy to overcome the rate-limiting steps of metastatic cascade through (a) enhanced interactions with protumorigenic myeloid cells and escape from T-dependent immune response mediated by CD8+ and natural killer (NK) cells; (b) production of immune mediators that establish a local and systemic tumor-supportive environment (premetastatic niche); (c) ability to survive either in the peripheral blood as circulating tumor cells (CTCs) or at the metastatic site forming a cooperative prometastatic loop with foreign "myeloid" cells, macrophages, and neutrophils, respectively. The development of cancer-specific "immune resistance" can be orchestrated either by cooperation with tumor microenvironment or by successive rounds of genetic/epigenetic changes. Recognition of the applicability of this model may provide effective therapeutic avenues for complete elimination of immune-resistant metastatic cells and for enhanced antitumor immunity as part of a combinatorial strategy.

\section{Introduction}

Metastasis remains the most significant cause of cancerassociated morbidity and mortality and specific targeting molecules have had limited success in reversing metastatic progression in the clinical setting [1-3]. Understanding the exact molecular and cellular basis of the events that facilitate cancer metastasis has been difficult so far. Over the past years, a well-accepted theory suggests that genomic alterations of the malignant cells accompanied by the so-called tumor microenvironment "nonmalignant cells" contribute to the metastatic cascade $[4,5]$. As such, metastasis is frequently described as the sequential execution of multiple steps. To establish the metastatic tumor, cancer cells have to acquire the traits that enable them to efficiently cooperate with the host stroma and simultaneously avoid antitumor immune response [4-9]. At early stage of carcinogenesis, tumors appear to be vulnerable because mutant and thus potentially immunogenic tumor cells are being exposed to the immune system, which can recognize them and restrict their growth $[10,11]$. This is the case of tumor-infiltrating immune cells particularly CD8+ T cells and NK cells which have the potential to restrict the tumor outgrowth or reject metastatic tumor cells $[12,13]$. According to this notion, in most primary tumors, a strong Th1/cytotoxic $\mathrm{T}$ cells infiltration correlates with a longer patient's survival [12-14]. Unfortunately, tumor develops multiple mechanisms of evading immune responses, by forming a compromised microenvironment that allows the dissemination of malignant cells in a foreign microenvironment through molecular mechanisms still 
poorly characterized. A variety of stromal cells, particularly M2-phenotype macrophages and myeloid-derived suppressor cells (MDSCs), are recruited to primary tumors; these not only enhance growth of the primary cancer but also facilitate its metastatic dissemination to distant organs $[13,14]$. Notably, cooperative "dialogue" between malignant cells and their microenvironment will go on in the systemic circulation and subsequently in the future metastatic site [13-17]. In fact, recent studies have demonstrated that a high systemic inflammatory response, that is, blood neutrophil-lymphocyte ratio (NLR), predicts lower overall survival, higher tumor stage, and a greater incidence of metastasis in multiple tumor types $[18,19]$. Therefore, a substantial amount of data suggests a novel dimension of the tumor biology and offers the opportunity to revisit the mechanisms describing evasion of cancer immunosurveillance during the metastatic process. The present review analyses recent studies that elucidate and reinforce the theory by which immune-phenotypic features or "immune resistance" by cancer cells may need to sustain the metastatic cascade and avoid antitumor immune response.

\section{Tumor Antigens and Antitumor Immune Response by Effectors of Adaptive Immunity}

A decade of studies has emphasized the nature of cancer as a systemic disease remarking a key role of host microenvironment as a critical hallmark. As a result, a new picture of cancer is emerging in particular due to unexpected crosstalk between malignant cells and the immune system [35]. Recent data have expanded the mechanisms of cancerimmune system interactions revealing that every known innate and adaptive immune effector component participates in tumor recognition and control $[9,10]$. It is now recognized that in different individuals and with different cancers, at early stage of tumorigenesis, the few cancer cells are detected by NK cells through their encounter with specific ligands on tumor cells [5]. In turn, activation of macrophages and dendritic cells and particularly $\mathrm{T}$ and $\mathrm{B}$ cells expands production of additional cytokines and further promotes activation of tumor-specific T cells "CD8+ cytotoxic T cells" leading to the generation of immune memory to specific tumor components [14-16]. However, in cases where the immune system is not able to eliminate the cancer, a state of equilibrium develops or eventually cancer cells can resist, avoid, or suppress the antitumor immune response, leading to the immune escape and a fully developed tumor (Figure 1) [9-15]. For example, investigations into the nature of cancer as a genetic disease have suggested two paradigmatic subtypes of colorectal cancer (CRC): chromosomal instability (CIN) and microsatellite instability (MSI), in which the expression of immunecheckpoint proteins can be differentially dysregulated to unleash the potential of the antitumor immune response [11]. In particular, tumors with mismatch-repair deficiency (dMMR) (10-20\%) of advanced colorectal cancer tend to have 10 to 100 times more somatic mutations and higher amount of lymphocyte infiltrates than mismatch-repairproficient colorectal cancers (pMMR), a finding consistent with a stronger antitumor immune response (Figure 1) [11, 20]. According to this notion, recent studies suggest that certain cancer subtypes dMMR CRC with high numbers of somatic mutations are more responsive to PD-1 blockade, a well-known immune-checkpoint inhibitor [20]. In particular, CD8-positive lymphoid infiltrate and membranous PDL1 expression on either tumor cells or tumor-infiltrating lymphocytes at the invasive fronts of the tumor are associated with an improved response to anti-PD-1 therapy in patients with mismatch-repair-deficient cancer $[11,20]$. In addition, cancer subtypes with stronger antitumor immune responses (immunogenic) are characterized by surface-exposed calreticulin or heat shock protein 90 (HSP90), which serve as a powerful mobilizing signal to the immune system in the context of damage-associated molecular patterns (DAMPs) [17]. As danger signals, DAMPs accompanied by subversion MHC Class I and II antigens on the plasma membrane of cancer cells appear to be characteristic of stressed or injured cells and can act as adjuvant signals to enhance antitumor immunity mediated by the innate immune system [17]. As described in this review, unfortunately, the large majority of human tumors can suppress the immune system to enhance their survival, rendering them invisible to cytotoxic T lymphocytes through a variety of mechanisms. Furthermore, in most cases, tumor-infiltrating immune cells differentiate into cells that promote each step of the tumor progression supporting ability of cancer cells to invade and survive in foreign organs. In addition, the intricate network of malignant and immune components represents a prominent obstacle to the effects of therapeutic agents.

\section{Tumor-Infiltrating Immune Cells and Immune Escape Mechanisms}

Tumors develop numerous strategies to avoid detection and eradication by the immune system. Among these, one of the best known includes the recruitment, expansion, and function of tumor-infiltrating leukocytes, such as immunoregulatory myeloid cells, that is, regulatory $\mathrm{T}$ cells (Treg cells) and $\mathrm{T}$ helper 17 cells (TH17 cells) [13-17]. In a variety of cancers, the transition from precancerous to invasive stage parallels a shift from Th1 to Th2 immune microenvironment. However, what makes the same $\mathrm{T}$ cell subset (e.g., CD8+ T cells) antitumorigenic in one cancer and protumorigenic in another remains largely unknown [13-17]. An increased number of $\mathrm{T}$ cells, including specifically activated CD8+ cytotoxic $\mathrm{T}$ cells (CTLs) and natural killer T (NKT) cells, generally correlate with better survival in some cancers as well as in patients with mismatch-repair-deficient colorectal cancer [13-17]. Accordingly, depletion of CD8+ T cells and NK cells consequently increases cancer metastasis without affecting primary tumor growth suggesting that cytotoxic lymphocytes have also metastasis-inhibiting effects [13]. The inflammatory contexts can promote tumor growth through the production of cytokines such as IL-6, IL-1, or TNF-a and angiogenic molecules such as VEGFA, placental growth factor (PlGF), or transforming growth factor-b (TGF-b) (Figure 2) [13-19, 22]. In addition, the so-called adenosinergic signaling cascade "adenosine production" mediated by CD39 and CD73 further promotes immune suppression as well as prostaglandin E2 

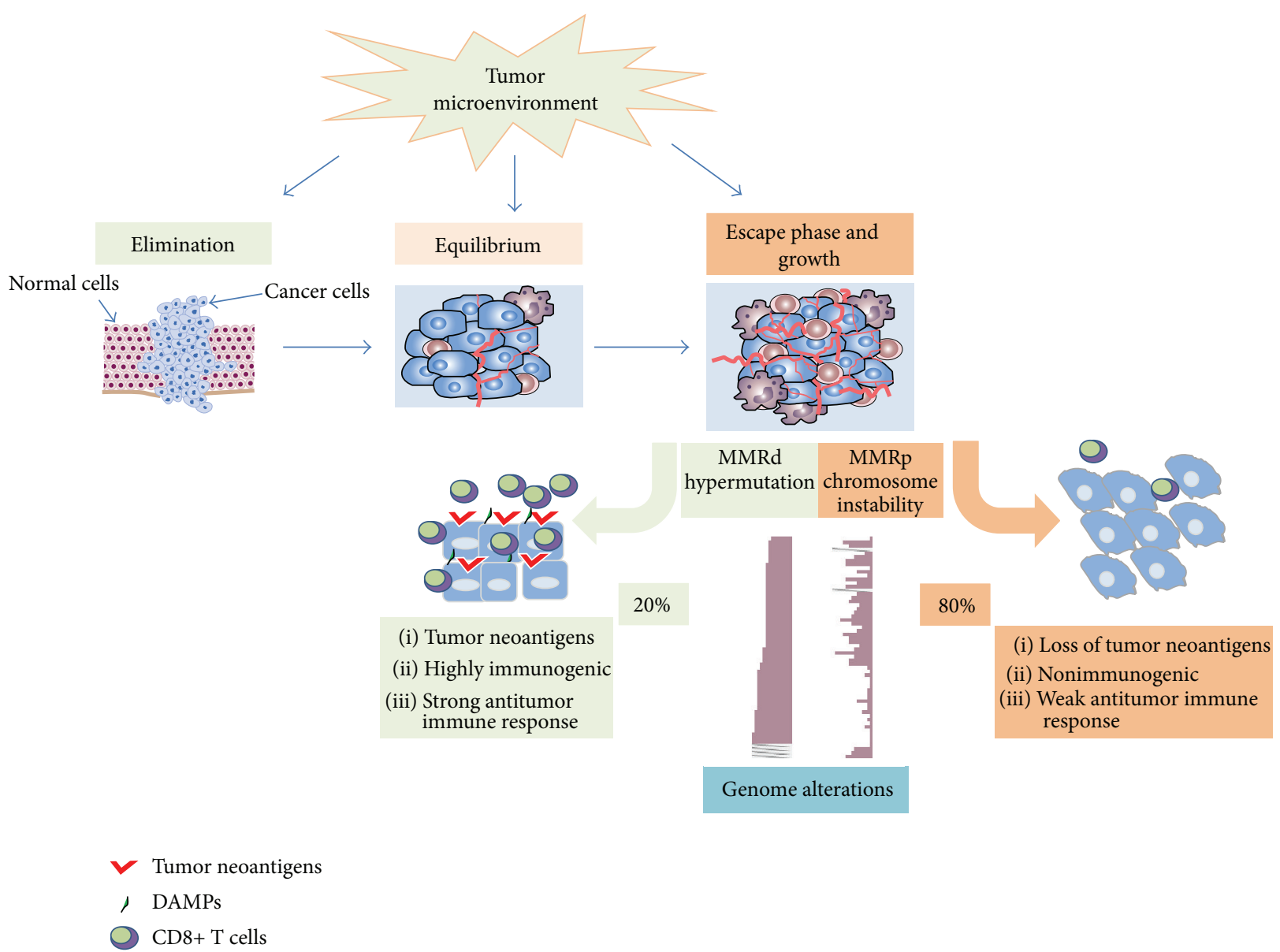

FIGURE 1: Tumor microenvironment and subversion of immunosurveillance during carcinogenesis. At early stage of primary tumor expansion, the immune system can specifically identify and eliminate tumor cells on the basis of their expression of specific antigens "highly immunogenic tumor." However, there might not be a complete elimination leading to the survival of some cancer cells that nevertheless remain under immunosurveillance "state of equilibrium." If the immune response fails to completely eliminate the tumor, cancer cells can avoid or suppress the antitumor immune response leading to a progressively growing tumor. In the case of colorectal cancer (CRC) subtypes, different combinations of genetic and epigenetic changes lead to well distinct microsatellite stable (MMRp) or unstable or MMRd subtypes with different mutational load. Studies have shown that MMRd CRC have a high number of somatic mutations, "hypermutated tumors," that can give rise to neoepitopes or damage-associated molecular patterns (DAMPs) and that these may serve as neoantigens for activation of tumor-specific CD8 T cell responses. The large majority of CRC are nonhypermutated cases tending to exhibit chromosomal instability associated with loss of tumor antigens, loss of human leukocyte antigen molecules, loss of sensitivity to complement, or T cell lysis, making them a poor target of an immune attack.

and suppressor myeloid or $\mathrm{T}$ cells attracting chemokines, including CXCL1, CXCL5, CCL2, and CCL12 [13, 14, 17]. This inflammatory milieu contributes to recruiting myeloid cells particularly the macrophages also named tumor associated macrophages (TAMs). They are recognized as the most common type of immune infiltrating cells protumorigenic in the tumor microenvironment (TME) in the primary tumors [15, 16]. Accumulating data suggest that TME polarizes recruited macrophages from a potentially tumor-reactive state (M1) to a tumor-promoting state (M2) phenotype [16]. TAMs can also exert prometastatic functions, by suppressing the cytotoxic activity of CD8+ T cells in primary tumors [13]. A type of immature myeloid cell that expresses CD11b and GR1 is also found in the TME. These cells can suppress the proliferation and the cytokine production of $\mathrm{T}$ cells in vitro and are thus referred to as myeloid-derived suppressor cells (MDSCs). Similar to macrophages, tumor associated neutrophils (TANs) with "N2 phenotype" can reduce CD8+ $\mathrm{T}$ cell activity and increase primary tumor growth (Figure 2). Interestingly, the prognostic role of elevated blood neutrophils and elevated blood neutrophil/lymphocyte ratio has been associated with poor clinical outcome [18, 19]. Instead, in the case of tumor-infiltrating neutrophils, recent studies suggest that high levels of myeloid cells CD33+HLA$\mathrm{DR}-\mathrm{CD} 16+$ are associated with improved colorectal cancer (CRC) prognosis [23-26]. Consistently, a high density of $\mathrm{MPO}+$ or CD15+ infiltrating cells consistent with the phenotype of the granulocytic lineage cells has been shown to be of benefit for CRC patient [26]. The role of neutrophil granulocytes (NGs) in the context of cancer-related inflammation remains matter of debate, even if their clinical relevance has recently begun to emerge. 


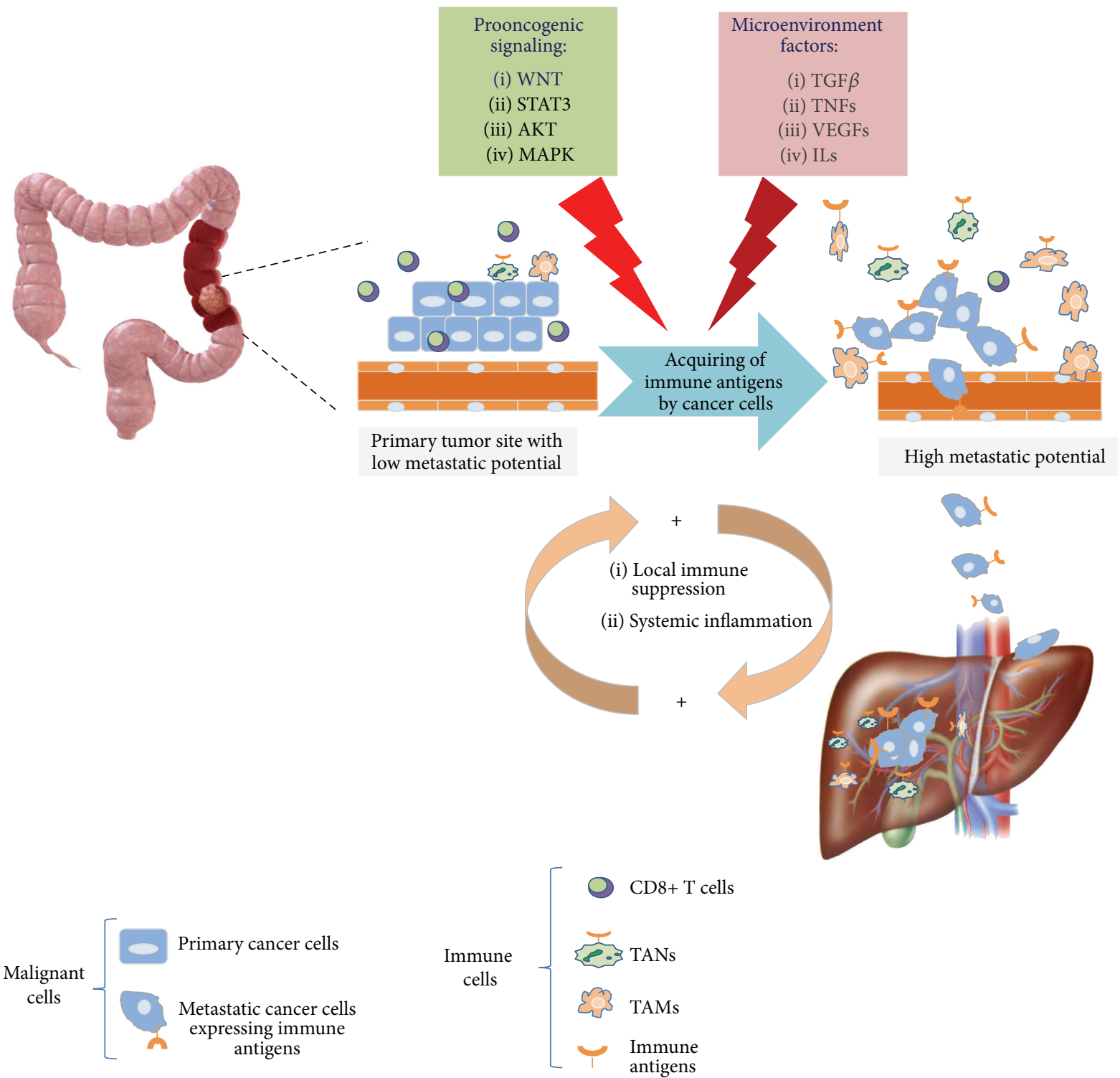

FIGURE 2: Immune antigens expression by cancer cells contributes to the metastatic cascade. Most malignant solid tumors metastasize from the primary organ to another, such as the liver. At the primary site, potentially immunogenic tumor cells are being exposed to the immune system. High density of TILs and tumoricidal immune response mediated by killer cells and CD8+ T cells restrict the tumor outgrowth. Genetic damage and deregulated signaling pathways accompanied by the production of various cytokines and chemokines in the tumor microenvironment can induce expression of immune antigens on the cancer cell surface. By these changes, the tumors promote recruitment and expansion of prometastatic myeloid cells, TAMs, or TANs, respectively. As such, immune-like phenotype enables cancer cells to increase metastatic potential and avoid immune surveillance at different levels during the metastatic cascade. The circulating tumor cells expressing an immune-like phenotype, that is, CD47, are then arrested in microvessels in the metastatic site where they need to survive. At the metastatic site, the arrested tumor cells escape from the resident myeloid cells and survive at the metastatic niche and proliferate to form the deadly metastatic tumor. Recent studies suggest that MAMs sustain the survival and persistent growth of emigrated cancer cells. TANs also promote the entrapment of circulating cancer cells by producing neutrophil extracellular traps (NETs). TAMs: tumor associated macrophages; MAMs: metastasis-associated macrophages; TANs: tumor associated neutrophils.

\section{Generation of a Tumor Microenvironment Permissive for Metastatic Cascade}

Once a cancer is established, the "dialogue" between malignant cells and their microenvironment progressively will go on up to reaching a cooperative interaction. As such, tumor-stromal interactions could help to avoid antitumor immune response and generate the so-called "tumor microenvironment permissive for metastasis" [13]. It is well known that the tumor activates certain immune-checkpoint pathways as a major mechanism of immune resistance, particularly against $\mathrm{T}$ cells that are specific for tumor antigens. By 


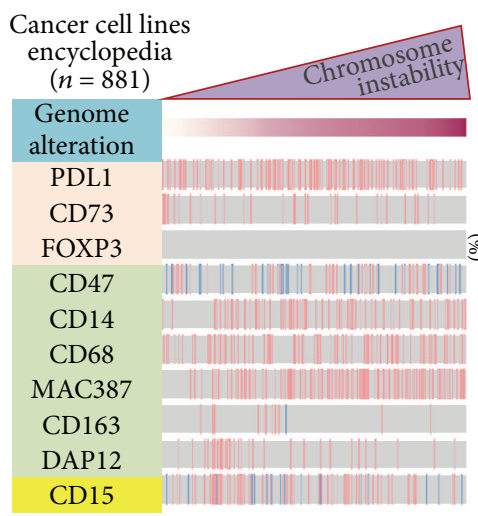

(a)

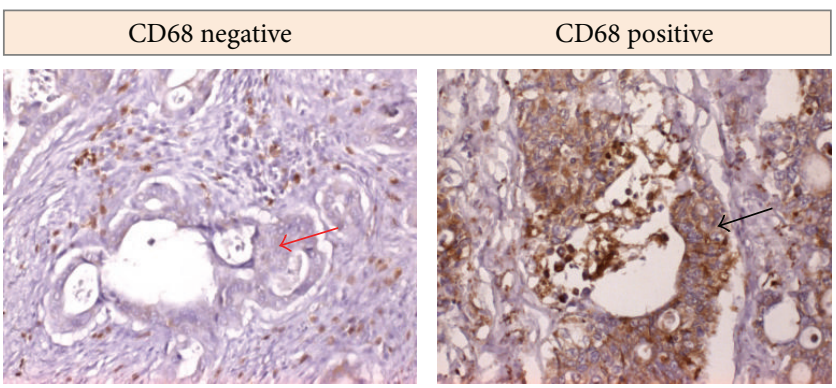

CD68 expression by tumor cells

(c)

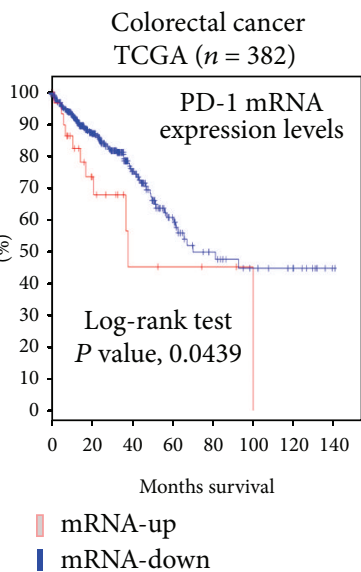

I mRNA-down

\begin{tabular}{|c|c|c|}
\hline $\begin{array}{l}\text { Cancer-related } \\
\text { expression }\end{array}$ & $\begin{array}{l}\text { Gene } \\
\text { name }\end{array}$ & Expression on immune cells \\
\hline $\begin{array}{l}\text { Breast, } \\
\text { colorectal, melanoma }\end{array}$ & PDL1 & Mainly myeloid linage, TILs \\
\hline Breast, ovarian, bladder & CD73 & $\mathrm{T}$ cells, B cells, nonmyeloid cells \\
\hline Colorectal & FOXP3 & TILs \\
\hline $\begin{array}{l}\text { Pancreas, colorectal, } \\
\text { CTC }\end{array}$ & $\mathrm{CD} 47$ & $\begin{array}{l}\text { Mainly Ms, less extended by NGs } \\
\text { Mainly Ms, less extended by NGs }\end{array}$ \\
\hline Bladder & CD14 & $\begin{array}{l}\text { DCs, B cells } \\
\text { Mainly Ms, less extended by }\end{array}$ \\
\hline Breast, colorectal & $\mathrm{CD} 68$ & nonmyeloid cells \\
\hline Breast & MAC387 & Mainly Ms, NGs \\
\hline Breast, colorectal & CD163 & Mainly Ms \\
\hline Breast & DAP12 & Mainly Ms \\
\hline Colorectal & CD15 & Mainly NGs, less extended by Ms \\
\hline
\end{tabular}

(b)

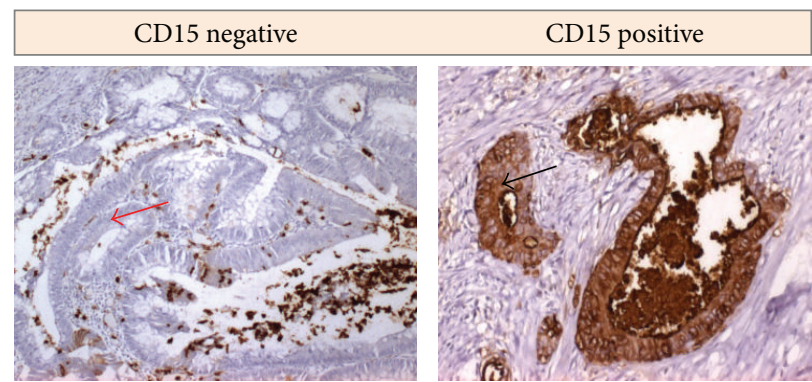

CD15 expression by tumor cells

(d)

FIGURE 3: Subsets of immune-phenotypic genes correlate with genome instability in a variety of tumors. (a) Genome instability represents one of the most important hallmarks of genetic diversity in tumors. We utilized a panel of 881 human tumor cell lines derived from different tissues of origin that has been extensively characterized for gene expression and copy-number variations and commonly used for genetic analysis and screening of potential chemotherapeutic agents [21]. mRNA expression of the immune antigens subdivided into low and high is shown. Kaplan-Meier curve shows that, compared with tumors expressing low PD-1 mRNA levels (blue line, 348/382, 91\%), tumors with high $P D-1$ mRNA expression (red line, 34/382, 9\%) are significantly associated with poor overall survival by interrogating the public colorectal cancer TCGA data set $(N=382)$. (b) Subsets of immune antigens and their prevalence in cancer cells in solid tumors are reported. (c, d) Immunohistochemical staining of colon cancer with neutrophils and macrophage antigens, CD15 and CD68, respectively. Black arrows indicate the immunostaining on the surface of malignant colonic cells. Red arrows indicate absence of immune antigen positivity, magnification 10x. TILs: tumor-infiltrating lymphocytes; Ms: macrophages; NGs: neutrophil granulocytes; CTCs: circulating tumor cells; DCs: dendritic cells.

upregulating cytotoxic T-lymphocyte-associated antigen 4 (CTLA4) and programmed cell death protein 1 (PD1), tumor cells block antitumor immune responses. Our unpublished observations indicate that tumors with high $P D-1$ mRNA expression are significantly associated with poor overall survival in colorectal cancer (Figure 3(a)). Therefore, actually, immune-checkpoint inhibitors against CTLA4 and PD1 are promising new approaches for tackling solid tumors [27-30]. In spite of this, the precise molecular mechanism by which cancer cells acquire the ability to suppress immune responses, evade immune system eradication, and contribute to the metastatic cascade remains still unknown. Numerous studies imply that genetic mutations of metastatic cancers do not significantly differ from their primary tumor [31]. Genomic profiling, second-generation sequencing, and proteomics have dramatically accelerated the effort to comprehensively characterize metastatic tumor cells and to understand their natural history of evolution from primary tumors. New observations have contributed to solidifying a mechanistic concept that a variety of immune-phenotypic antigens can be expressed on the tumor cell surface in many solid tumors (Figures 3(a)-3(d)) [21, 32-39]. Acquisition of such a characteristic by cancer cells is generally correlated to early distant recurrence, local recurrence, and reduced survival time in several tumors, that is, breast, colorectal, and pancreas [21, 32-39]. Thus, cancer-immune-like phenotype in the TME might be relevant to enhance the cooperation with prometastatic immune cells either by contact or by the production of paracrine/autocrine immune-suppressive mediators (Figure 2). This aberrant phenotype may simultaneously inhibit antitumor immune responses and promote metastatic disease at different levels. 


\section{Tumor Immune Resistance: A Model of Metastatic Immune Escape}

Over 100 years ago, the English surgeon Stephen Paget described the "seed and soil" theory of metastasis likening tumor cells to "seeds" that are systemically distributed and able to only inhabit particular microenvironment, the "soils." In the last years, we have begun to reveal fundamental concepts that sustain Paget's hypothesis, pointing to the existence of strategies whereby cancer cells evade immune effectors.

The discovery of effective metastasis-targeting agents (immune-checkpoint inhibitors) that specifically interrupt the communication between cancer cells and their microenvironment has the potential to produce durable clinical responses [30]. Then, how does cancer cell disseminate in several foreign microenvironments? Cancer cells employ several strategies to escape their site of origin and survive in potentially hostile microenvironments, many of which remain still poorly understood. Recent studies revealed that the pattern of the tumor microenvironment remains a major prognostic factor even in the metastatic lesions. Tumor-infiltrating immune cells differentiate into cells that promote each step of the metastatic cascade. In particular, myeloid cells such as tumor associated macrophages (TAMs) and tumor associated neutrophils (TANs) contribute to establishing and maintaining metastatic foci. Several environmental factors, including interleukin-4 (IL-4), tumor-derived transforming growth factor- $\beta(\operatorname{TGF} \beta)$, and macrophage migration inhibitory factor (MIF), create a permissive tumor microenvironment for metastasis (TMEM) where the cancer cells intravasate $[21,30$, 36].

Further lines of evidence support the role of genome instability, which generates the genetic diversity and consequently can expedite the acquisition of a repertoire of macrophage, neutrophil, or T lymphocyte antigens by cancer cells (Figures 1 and 3(a)) [21, 30, 36]. Multiple general mechanisms are emerging. (a) constitutive oncogenic signaling can upregulate expression of immune genes on tumor cell surface, independently of TME. For example, activation of the AKT and signal transducer and activator of transcription 3 (STAT3) pathways has been reported to drive PDL1 expression, which inhibits local antitumor $\mathrm{T}$ cell-mediated responses by direct contact with PD1. This latter is expressed on a large proportion of tumor-infiltrating lymphocytes (TILs); high expression levels of PDL1 on either tumor cells or TILs associate with poor prognosis relative to PDL1 negative tumors [30]. In colorectal carcinoma, membranous PDL1 expression correlates with CD8-positive lymphoid infiltrate located at the invasive fronts of the tumor only in patients with mismatch-repair-deficient cancer. However, expression of CD8 and PD-L1 was not significantly associated with progression-free survival or overall survival [20], suggesting that further studies are needed to explain such results. (b) Prooncogenic signaling can also alter the balance between ATP/ADP, AMP, and adenosine, crucial for tumor progression. The best-understood mechanism includes overexpression of CD73 on cancer cells that plays a crucial role in the adenosinergic signaling by producing adenosine, one of the most important immunosuppressive regulatory molecules in the TME [39]. (c) Alternatively, upregulation of immune antigens is induced on tumor cells in response to cytokines such as interferons (IFNs) following an adaptive response to endogenous antitumor immunity (Figure 2) [39]. At the primary tumor site, these mechanisms allow cancer cells to resist immune elimination and might represent an alternative to the conventional drug resistance mechanisms that involve the mutation of drug targets. However, recent studies also suggest that ectopic immune antigens expressed on the cell surface of malignant cells may help to overcome the rate-limiting steps of metastasis including the need to survive in the blood circulation either as "circulating tumor cells" (CTCs) or by facilitating "retention" at distant metastatic sites [37]. The process of extravasation, retention, and persistent growth of emigrated cancer cells may be supported by direct interactions with the so-called metastasis-associated macrophages (MAMs) or resident neutrophils in foreign organs (Figure 2). Indeed, transcriptomic analyses from CTCs have revealed a marked overexpression of CD47, which is recognized to be a specific macrophage antigen [37]. Thus, it has been proposed that the "do not eat me" signal on cancer cells communicates to the signal regulatory protein- $\alpha$ on macrophages and cytotoxic T lymphocytes and prevents their phagocytosis. In addition, expression of nonimmunogenic phenotype by CTC could help to overcome the hostile blood stream environment for tumor cells during the metastatic dissemination [37]. Macrophage traits in tumor cells appear to be the most common and include the expression of the following antigens: CD14, CD68, MAC387, CD163, and DAP12 (Figures 3(b) and $3(c))$. However, immune-phenotypic features of the tumor cells are not restricted to the macrophage lineage. To shed light on these aspects, we have recently investigated the clinical significance of a series of immune-phenotypic markers expressed on malignant cells from metastatic CRC patients receiving first-line therapy with targeting agents cetuximab and bevacizumab, respectively [40]. Strikingly, not only did this approach confirm previous links with macrophages antigens, but it also revealed an unexpected connection with NG antigen CD15 protein encoded by FUT4 gene (Figure 3(d)) $[40,41]$. In fact, increased expression of CD15 on tumor cells reflected low levels of intratumoral CD8+ TILs and high systemic inflammation and predicted poorer outcomes in terms of progression-free survival. According to this finding, CD15 was consistently higher in pMMR than in dMMR colorectal cancer. The subsequent bioinformatic prediction was intriguing, because it revealed that CD15 overexpression by tumor cells is subjected to immune resistance as driven by constitutive oncogenic RAF-MEK-ERK kinase signaling pathways through involvement of prooncogenic receptors $E R B B 3$ or HER3 and FGFR4 activation [40]. In line with this, ERBB3 acts as a major cause of treatment failure in cancer therapy, mainly through activation of the PI-3 K/AKT, MEK/ MAPK, and Jak/Stat signaling pathways [42]. The molecular mechanisms underlying expression of immune antigens in cancers remain still poorly understood. We cannot exclude the contribution of genetic exchange mediated-cell fusion or genetic exchange between the cells by exosome-mediated transfer. The neutralization of signals through these ectopic antigens might enhance $\mathrm{T}$ cell responses to eliminate tumor 
cells. Another possible therapeutic strategy is withdrawal of microenvironmental support for metastatic cancer cells by targeting prometastatic immune cells, in particular macrophages. Thus, a more detailed understanding of the effector mechanisms and the essential cellular interactions between the various cell types in the TME is needed to allow for more precise therapeutic intervention.

\section{Concluding Remarks}

Over the past decade, the scenario of metastatic process is changing. Studies on the molecular profiles of tumors and tumor associated cells imply that metastatic potential becomes an active process at early stage in cancer progression. Current results indicate that tumors predominantly recruit specific types of suppressor cells to evade antimetastatic immune responses. For metastasis to occur from solid malignancies, tumor cells need to undergo a series of successive rounds of genetic/epigenetic mutation and selection. As such, a key step in the initiation of metastatic cascade may be the selection of nonimmunogenic tumor cell variants in the primary tumor. The data discussed in this review seek to consolidate a new scenario of the tumor biology, in which aberrant immune-phenotypic features expressed by cancer cells contribute to the metastatic cascade evading antitumor immune response. This putative "immune-like phenotype" might be imposed by persistent genetic/epigenetic alterations of the cancer cells and orchestrated by prometastatic function of nonmalignant infiltrating cells. As such cancer-specific immunosubversion protects emigrated cancer cells from surveillance by killer cells and permits the recruitment of immunosuppressive cells in foreign microenvironments through "do not eat me signals" or the so-called homotypic direct or indirect interactions. Detailed knowledge underlying these processes might have important implications to shed light on the impact of tumor heterogeneity on cancer outcomes. They might provide novel platforms to the design of novel immunotherapies aimed at properly activating $\mathrm{T}$ cells or modulating inflammatory and immunosuppressive elements. Recognition of the widespread applicability of these concepts could be of help either in the definition of surrogate immune-checkpoint biomarkers that dominate in a particular tumor or as guide for the choice of personalized immune inhibitor molecules.

\section{Conflict of Interests}

The authors declare that there is no conflict of interests regarding the publication of this paper.

\section{Authors' Contribution}

Pietro Parcesepe and Guido Giordano contributed equally to this work.

\section{References}

[1] J. Gagan and E. M. Van Allen, "Next-generation sequencing to guide cancer therapy," Genome Medicine, vol. 7, no. 1, article 80, 2015.
[2] A. Bardelli and S. Siena, "Molecular mechanisms of resistance to cetuximab and panitumumab in colorectal cancer," Journal of Clinical Oncology, vol. 28, no. 7, pp. 1254-1261, 2010.

[3] S. Valastyan and R. A. Weinberg, "Tumor metastasis: molecular insights and evolving paradigms," Cell, vol. 147, no. 2, pp. 275292, 2011.

[4] A. J. Gentles, A. M. Newman, C. L. Liu et al., "The prognostic landscape of genes and infiltrating immune cells across human cancers," Nature Medicine, vol. 21, no. 8, pp. 938-945, 2015.

[5] A. Mantovani, P. Allavena, A. Sica, and F. Balkwill, "Cancerrelated inflammation," Nature, vol. 454, no. 7203, pp. 436-444, 2008.

[6] D. G. DeNardo, M. Johansson, and L. M. Coussens, "Immune cells as mediators of solid tumor metastasis," Cancer and Metastasis Reviews, vol. 27, no. 1, pp. 11-18, 2008.

[7] J. Condeelis and J. W. Pollard, "Macrophages: obligate partners for tumor cell migration, invasion, and metastasis," Cell, vol. 124, no. 2, pp. 263-266, 2006.

[8] O. Trédan, C. M. Galmarini, K. Patel, and I. F. Tannock, "Drug resistance and the solid tumor microenvironment," Journal of the National Cancer Institute, vol. 99, no. 19, pp. 1441-1454, 2007.

[9] I. P. Witz, "The tumor microenvironment: the making of a paradigm," Cancer Microenvironment, vol. 2, supplement 1, pp. S9-S17, 2009.

[10] N. Sethi and Y. Kang, "Unravelling the complexity of metastasis-molecular understanding and targeted therapies," Nature Reviews Cancer, vol. 11, no. 10, pp. 735-748, 2011.

[11] B. B. McConnell and V. W. Yang, "The role of inflammation in the pathogenesis of colorectal cancer," Current Colorectal Cancer Reports, vol. 5, no. 2, pp. 69-74, 2009.

[12] H. A. Smith and Y. Kang, "The metastasis-promoting roles of tumor-associated immune cells," Journal of Molecular Medicine, vol. 91, no. 4, pp. 411-429, 2013.

[13] T. Kitamura, B.-Z. Qian, and J. W. Pollard, "Immune cell promotion of metastasis," Nature Reviews Immunology, vol. 15, no. 2, pp. 73-86, 2015.

[14] M. Pancione, G. Giordano, A. Remo et al., "Immune escape mechanisms in colorectal cancer pathogenesis and liver metastasis," Journal of Immunology Research, vol. 2014, Article ID 686879, 11 pages, 2014.

[15] M. De Palma and C. E. Lewis, "Macrophage regulation of tumor responses to anticancer therapies," Cancer Cell, vol. 23, no. 3, pp. 277-286, 2013.

[16] A. Mantovani, T. Schioppa, C. Porta, P. Allavena, and A. Sica, "Role of tumor-associated macrophages in tumor progression and invasion," Cancer and Metastasis Reviews, vol. 25, no. 3, pp. 315-322, 2006.

[17] D. V. Krysko, A. D. Garg, A. Kaczmarek, O. Krysko, P. Agostinis, and P. Vandenabeele, "Immunogenic cell death and DAMPs in cancer therapy," Nature Reviews Cancer, vol. 12, no. 12, pp. 860875, 2012.

[18] G. Malietzis, M. Giacometti, R. H. Kennedy, T. Athanasiou, O. Aziz, and J. T. Jenkins, "The emerging role of neutrophil to lymphocyte ratio in determining colorectal cancer treatment outcomes: a systematic review and meta-analysis," Annals of Surgical Oncology, vol. 21, no. 12, pp. 3938-3946, 2014.

[19] D. C. McMillan, "Systemic inflammation, nutritional status and survival in patients with cancer," Current Opinion in Clinical Nutrition \& Metabolic Care, vol. 12, no. 3, pp. 223-226, 2009.

[20] D. T. Le, J. N. Uram, H. Wang et al., "PD-1 blockade in tumors with mismatch-repair deficiency," The New England Journal of Medicine, vol. 372, no. 26, pp. 2509-2520, 2015. 
[21] J. Barretina, G. Caponigro, N. Stransky et al., "The Cancer Cell Line Encyclopedia enables predictive modelling of anticancer drug sensitivity," Nature, vol. 483, no. 7391, pp. 603-607, 2012.

[22] G. Giordano, A. Febbraro, M. Venditti et al., "Targeting angiogenesis and tumor microenvironment in metastatic colorectal cancer: role of aflibercept," Gastroenterology Research and Practice, vol. 2014, Article ID 526178, 13 pages, 2014.

[23] N. A. Giraldo, E. Becht, R. Remark, D. Damotte, C. SautèsFridman, and W. H. Fridman, "The immune contexture of primary and metastatic human tumours," Current Opinion in Immunology, vol. 27, no. 1, pp. 8-15, 2014.

[24] J. P. Väyrynen, A. Tuomisto, K. Klintrup, J. Mäkelä, T. J. Karttunen, and M. J. Mäkinen, "Detailed analysis of inflammatory cell infiltration in colorectal cancer," British Journal of Cancer, vol. 109, no. 7, pp. 1839-1847, 2013.

[25] F. Donskov, "Immunomonitoring and prognostic relevance of neutrophils in clinical trials," Seminars in Cancer Biology, vol. 23, no. 3, pp. 200-207, 2013.

[26] R. A. Droeser, C. Hirt, S. Eppenberger-Castori et al., "High myeloperoxidase positive cell infiltration in colorectal cancer is an independent favorable prognostic factor," PLoS ONE, vol. 8, no. 5, Article ID e64814, 2013.

[27] C. Tecchio, P. Scapini, G. Pizzolo, and M. A. Cassatella, "On the cytokines produced by human neutrophils in tumors," Seminars in Cancer Biology, vol. 23, no. 3, pp. 159-170, 2013.

[28] A. Snyder, V. Makarov, T. Merghoub et al., "Genetic basis for clinical response to CTLA-4 blockade in melanoma," The New England Journal of Medicine, vol. 371, no. 23, pp. 2189-2199, 2014.

[29] M. Yadav, S. Jhunjhunwala, Q. T. Phung et al., "Predicting immunogenic tumour mutations by combining mass spectrometry and exome sequencing," Nature, vol. 515, no. 7528, pp. 572576, 2014.

[30] S. L. Topalian, C. G. Drake, and D. M. Pardoll, "Immune checkpoint blockade: a common denominator approach to cancer therapy," Cancer Cell, vol. 27, no. 4, pp. 450-461, 2015.

[31] J. A. Joyce and J. W. Pollard, "Microenvironmental regulation of metastasis," Nature Reviews Cancer, vol. 9, no. 4, pp. 239-252, 2009.

[32] L. Larizza, V. Schirrmacher, and E. Pfluger, "Acquisition of high metastatic capacity after in vitro fusion of a nonmetastatic tumor line with a bone marrow-derived macrophage," Journal of Experimental Medicine, vol. 160, no. 5, pp. 1579-1584, 1984.

[33] M. Munzarova, L. Lauerova, and J. Capkova, "Are advanced malignant melanoma cells hybrids between melanocytes and macrophages?" Melanoma Research, vol. 2, no. 2, pp. 127-129, 1992.

[34] M. B. Maniecki, A. Etzerodt, B. P. Ulhøi et al., "Tumorpromoting macrophages induce the expression of the macrophage-specific receptor CD163 in malignant cells," International Journal of Cancer, vol. 131, no. 10, pp. 2320-2331, 2012.

[35] I. Shabo, H. Olsson, X.-F. Sun, and J. Svanvik, "Expression of the macrophage antigen CD163 in rectal cancer cells is associated with early local recurrence and reduced survival time," International Journal of Cancer, vol. 125, no. 8, pp. 18261831, 2009.

[36] I. Shabo and J. Svanvik, "Expression of macrophage antigens by tumor cells," in Cell Fusion in Health and Disease, vol. 950 of Advances in Experimental Medicine and Biology, pp. 141-150, Springer, Dordrecht, The Netherlands, 2011.

[37] G. Steinert, S. Schölch, T. Niemietz et al., "Immune escape and survival mechanisms in circulating tumor cells of colorectal cancer," Cancer Research, vol. 74, no. 6, pp. 1694-1704, 2014.
[38] T. Grimmig, M. Kim, C.-T. Germer, M. Gasser, and A. M. Waaga-Gasser, "The role of FOXP3 in disease progression in colorectal cancer patients," OncoImmunology, vol. 2, no. 6, Article ID e24521, 2013.

[39] J. Stagg, "The double-edge sword effect of anti-CD73 cancer therapy," OncoImmunology, vol. 1, no. 2, pp. 217-218, 2012.

[40] G. Giordano, A. Febbraro, E. Tomaselli et al., "Cancer-related CD15/FUT4 overexpression decreases benefit to agents targeting EGFR or VEGF acting as a novel RAF-MEK-ERK kinase downstream regulator in metastatic colorectal cancer," Journal of Experimental \& Clinical Cancer Research, vol. 34, article 108, 2015.

[41] D. I. Gabrilovich, S. Ostrand-Rosenberg, and V. Bronte, "Coordinated regulation of myeloid cells by tumours," Nature Reviews Immunology, vol. 12, no. 4, pp. 253-268, 2012.

[42] C. Sun, S. Hobor, A. Bertotti et al., "Intrinsic resistance to MEK inhibition in kras mutant lung and colon cancer through transcriptional induction of ERBB3," Cell Reports, vol. 7, no. 1, pp. 86-93, 2014. 


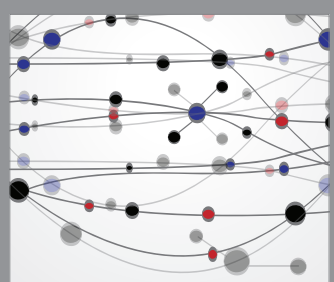

The Scientific World Journal
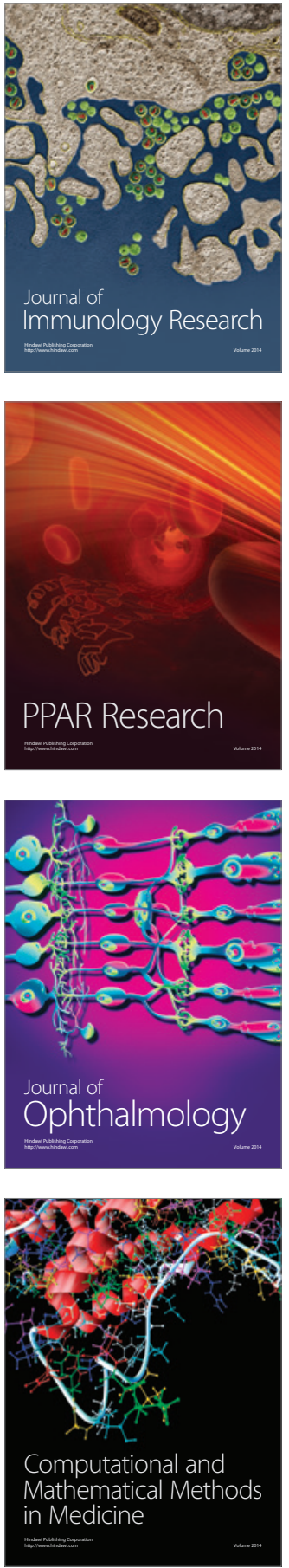

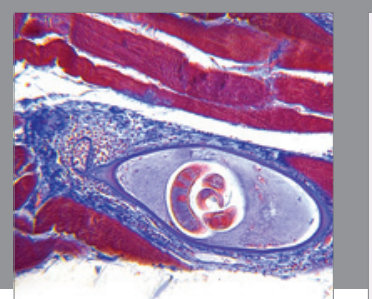

Gastroenterology Research and Practice

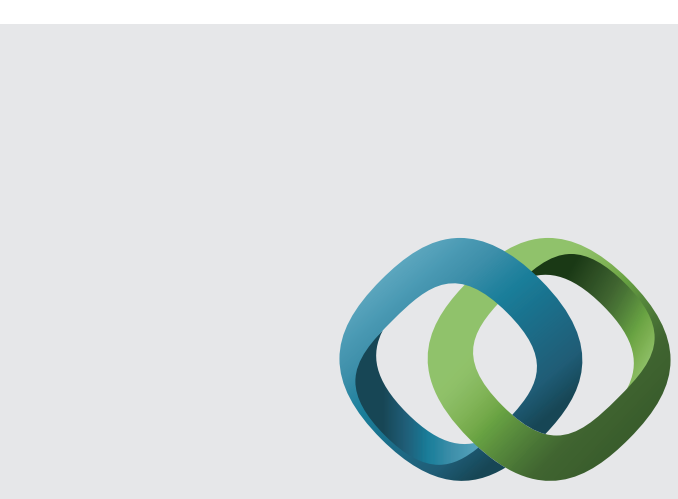

\section{Hindawi}

Submit your manuscripts at

http://www.hindawi.com
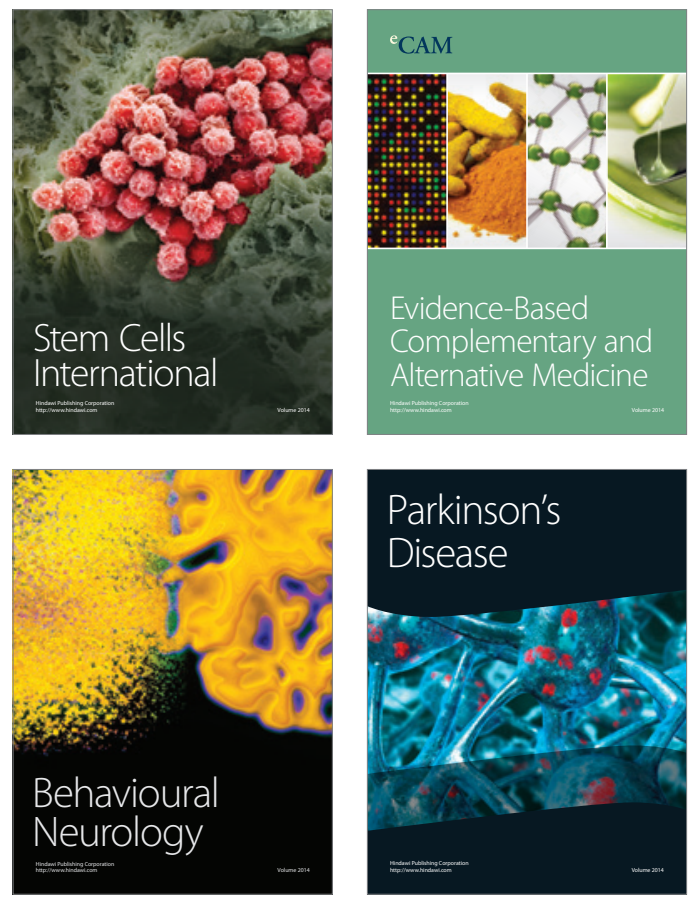
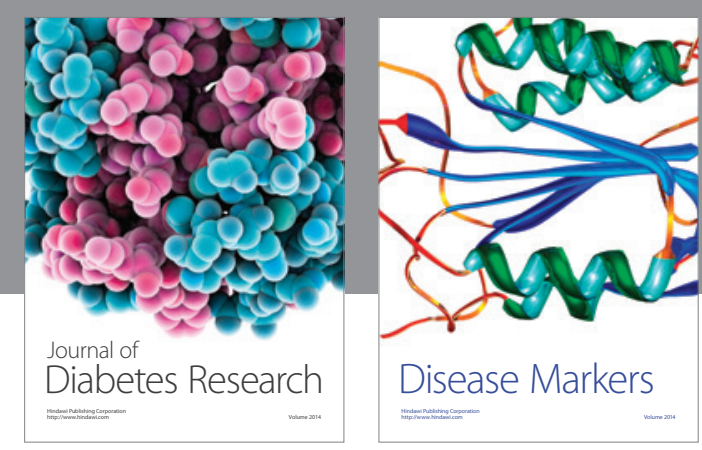

Disease Markers
